TRANSACTIONS OF THE

AMERICAN MATHEMATICAL SOCIETY

Volume 317, Number 1, January 1990

\title{
EXTENSIONS OF VALUATION RINGS IN CENTRAL SIMPLE ALGEBRAS
}

\author{
H. H. BRUNGS AND J. GRÄTER
}

\begin{abstract}
Certain subrings $R$ of simple algebras $Q$, finite dimensional over their center $K$, are studied. These rings are called $Q$-valuation rings since they share many properties with commutative valuation rings. Let $V$ be a valuation ring of $K$, the center of $Q$, and let $\mathscr{R}$ be the set of $Q$-valuation rings $R$ in $Q$ with $R \cap K=V$, then $|\mathscr{R}| \geq 1$. This extension theorem, which does not hold if one considers only total valuation rings, was proved by N. I. Dubrovin. Here, first a somewhat different proof of this result is given and then information about the set $\mathscr{R}$ is obtained. Theorem. The elements in $\mathscr{R}$ are conjugate if $V$ has finite rank. Theorem. The elements in $\mathscr{R}$ are total valuation rings if $\mathscr{R}$ contains one total valuation ring. In this case $Q$ is a division ring. Theorem. $|\Re|=1$ if $\mathscr{R}$ contains an invariant total valuation ring.
\end{abstract}

Any valuation $v$ (in the sense of Krull [17]) on a commutative field $K$ has an extension to an arbitrary extension field $F$. If $V$ is the valuation ring of $K$ correspondong to $v$ this means that there exists a valuation ring $B$ of $F$ with $B \cap K=V$. If, in addition, $F$ is a Galois extension of $K$ then $\{\sigma(B) \mid \sigma \in G(F / K)\}$ is exactly the set of valuation rings of $F$ that intersect with $K$ in $V$, where $G(F / K)$ is the Galois group of $F$ over $K$ [11].

In order to consider an analogous noncommutative situation one must decide on what it means to have a valuation on a division ring. Schilling [25] extended Krull's definition as follows: A valuation $v$ on a division ring $D$ is a mapping $v$ from $D^{*}=D \backslash 0$ onto an ordered group such that $v(a b)=v(a) \circ v(b)$ and $v(a+b) \geq \min \{v(a), v(b)\}$ for $a, b$ in $D^{*}$ (see also [26]).

Such valuations on $D$ correspond to subrings $B$ of $D$ with the following two properties:

(T) If $x$ is in $D, x$ not in $B$ then $x^{-1}$ is in $B$; we say $B$ is a total valuation ring of $D$.

(I) $d B d^{-1}=B$ for all $d \neq 0$ in $D$, i.e. $B$ is invariant.

Received by the editors June 14, 1988

1980 Mathematics Subject Classification (1985 Revision). Primary 16A40, 16A39, 16A10, 16A14, 16A16; Secondary 12E15, 12J20, 13A18, 13B02, $13 \mathrm{~B} 05$.

Key words and phrases. Valuation ring, simple artinian algebra, division ring, extensions, completions, Galois theory, localization, Bezout order.

The first author is supported in part by NSERC. 
One can then consider the problem of describing the set of all invariant total valuation rings $B$ in the division ring $D$ with $B \cap K=V$ where $V$ is a given valuation ring in the center $K$ of $D$ and $[D: K]<\infty$. The example of the quaternions $H$ over the rationals shows that such an extension (even if it is not invariant) does not exist in general (only the 2-adic valuation can be extended) and Wadsworth in [28], generalizing a result by Cohn [8], showed that at most one such $B$ exists.

It was proved in [5] that total valuation rings in $D$ that extend the same $V$ are conjugate and that their number is $\leq \sqrt{[D: K]}$. Total valuation rings were also studied by Mathiak [20, 21].

Commutative valuation rings can be described as the elements with finite values under a place mapping. Dubrovin in [9], replacing the fields by simple artinian algebras, gives the following definition: A subring $R$ of a simple artinian algebra $Q$ is a $Q$-valuation ring if $R$ contains an ideal $M$ such that $R / M$ is simple artinian and for each element $q$ in $Q \backslash R$ there exist elements $r_{1}, r_{2}$ in $R$ with $r_{1} q, q r_{2}$ in $R \backslash M$.

A $D$-valuation ring $R$, where $D$ is a division ring, is a total valuation ring of $D$ if and only if $R / M$ is a division ring (Lemma 2.2). In $\S 2$ of the present paper we will recall some facts about $Q$-valuation rings and prove in $\S 3$ the following

Theorem (Dubrovin). For every valuation ring $V$ in the center $K$ of a simple algebra $Q$, finite dimensional over $K$, there exists a $Q$-valuation ring $R$ with $R \cap K=V$.

The proof given here will avoid the transfinite argument used in [10].

The final two sections deal with the set $\mathscr{R}$ of $Q$-valuation rings that extend a valuation ring in the center $K$ of a finite dimensional simple algebra $Q$. It is proved in $\S 5$ that the elements in $\mathscr{R}$ are conjugate in $Q$ if $V$ has finite rank. If $V$ has rank 1 a real norm function is introduced in $Q$, that extends the given valuation on $V$ and leads to a completion of $Q$. In $\S 4$ it is shown that $|\mathscr{R}|=1$ (and $Q$ is a division ring) if $\mathscr{R}$ contains an invariant total valuation ring. All elements in $\mathscr{R}$ are total if one is total.

Dubrovin's extension theorem does not fully generalize the commutative theorem cited at the beginning. Even the condition that $K$ is in the center of $Q$ is necessary: No valuation ring in $\mathbb{Q}(i)$ can be extended by an $H$-valuation ring in $H$ except the extension of the 2-adic valuation.

Finally, we mention two related results. Cohn and Mahdavi-Hezavehi in [7] consider invariant total valuations with abelian value groups and give conditions for such extensions to be possible. In [4] conditions on the chain ring $R_{0}$, with monomorphism $\sigma$, and $\sigma$-derivation $\delta$ are given that ensure that a total valuation ring $R$ exists in the Ore extension $D(x, \sigma, \delta)$, with $R_{0}=R \cap D$, where $D$ is the skewfield of quotients of $R_{0}$. 
Rings that can be considered as the noncommutative equivalent of commutative valuation rings occur for example as coordinate rings of $\mathrm{Hjelmslev}$ planes [16], as localizations of rings with a distributive lattice of right ideals [3, 13], in the construction of division rings $[1,6,15,19,22,27]$ or as components in structure theorems for certain classes of rings [12, 23].

Rings $R$ in the above list are all right chain rings with possibly zero divisors, i.e. for $a, b$ in $R$ either $a R \subseteq b R$ or $b R \subset a R$ holds. A ring that is a right as well as a left chain ring is called a chain ring. The total valuation rings defined in section 1 are exactly the chain domains. A ring is called right invariant if all its right ideals are two sided and invariant if it is right and left invariant.

Even though extensions of valuations are of central importance in the commutative case, no general extension theory exists for noncommutative valuations. The problem can be (somewhat loosely) formulated as follows: Let $\mathscr{V}$ be a class of rings that serve as noncommutative valuation rings. For example, the class of right chain rings, of total valuation rings, of invariant total valuation rings, of abelian or real invariant total valuation rings. We assume that for every $R$ in $\mathscr{V}$ there exists a certain ring $Q(R)$ of fractions such that $Q(R)$ is in a class $\mathscr{F}$ consisting of the rings $T$ with $Q(T)=T$.

A ring $R$ in $\mathscr{V}$ is called an extension in $T$ of $R_{0}$ in $\mathscr{V}$ if $T$ is in $\mathscr{F}$ with $T=Q(R) \supseteq Q\left(R_{0}\right)$ and $R \cap Q\left(R_{0}\right)=R_{0}$.

Problem. For a given $R_{0}$ and $T$ describe the set of all extensions of $R_{0}$ in $T$.

Generalizing the notion of right chain rings to that of right $n$-chain rings $R$ in an overring $Q$-i.e. for any set of $n+1$ elements $a_{0}, \ldots, a_{n}$ in $Q$ there exists a $j$ with $a_{j} R \subseteq \sum a_{i} R, i \neq j$-Dubrovin [9] arrives at the following definition of a $Q$-valuation ring, which defines a class of rings which is closed under Morita equivalence and rich enough to allow a very interesting extension theory.

Definition 2.1. A subring $R$ of a simple artinian algebra $Q$ is called a $Q$ valuation ring if $R$ contains an ideal $M$ such that $R / M$ is simple artinian and for any element $q$ in $Q \backslash R$ there exist $r_{1}, r_{2}$ in $R$ with $r_{1} q, q r_{2}$ in $R \backslash M$.

Matrix rings over chain domains, Azumaya algebras over commutative valuation rings [10] and localizations of noncommutative prime Dedekind rings [18] provide examples for $Q$-valuation rings. A particular example is the ring $R=\mathbb{Z}_{3}+\mathbb{Z}_{3} i+\mathbb{Z}_{3} j+\mathbb{Z}_{3} k$ in $H$, the quaternions over the rationals, which extends the 3 -adic valuation ring $\mathbb{Z}_{3}$ in $\mathbb{Q}$.

Lemma 2.2. Let $R$ be a $Q$-valuation ring with $Q$ and $R / M$ division rings. Then $R$ is a total valuation ring.

Proof. Assume that $x$ is in $Q \backslash R$. Then an element $r$ in $R$ exists with $x r$ in $R \backslash M$, and $x r r^{\prime}=1+m$ for some $r^{\prime}$ in $R, m$ in $M$ follows. The element $(1+m)^{-1}$ is in $R$ since otherwise $\left((1+m)^{-1}-1\right) s=u$ is in $R \backslash M$ for some 
$s$ in $R$. Then, $(1+m)\left((1+m)^{-1}-1\right) s=-m s$ is in $M$; but this element is also equal to $(1+m) u=u+m u$ in $R \backslash M$, a contradiction. It follows that $x^{-1}$ is in $R$ and $R$ is total.

We will now collect some results from [9 and 10] that will be used in the following sections.

Let $R$ be a $Q$-valuation ring as in Definition 2.1. It follows from [9] that then $M=J(R)$ (the Jacobson radical of $R$ ), that every finitely generated right or left ideal in $R$ is principal (i.e. $R$ is a Bezout ring) and that $R$ is an order in $Q$.

The converse also holds:

Theorem 2.3 [9]. The subring $R$ of the simple artinian algebra $Q$ is a $Q$ valuation ring if and only if $R / J(R)$ is simple artinian and $R$ is a Bezout order of $Q$.

It follows that a right chain ring $R$ which is a $Q$-valuation ring is also a domain, since otherwise the set $I$ of elements in $R$ with nonzero left annihilator is an ideal of $R$ consisting of zero divisors only. In that case $Q$ is a division ring and $R$ is a chain domain, i.e. a total valuation ring, since $R$ is a right and left order in a division ring.

Every simple artinian algebra $Q$ is isomorphic to a matrix ring $(D)_{n}$ for a division ring $D$ and some $n$ (see [24]). The next result describes the $(D)_{n}$ valuation rings as being isomorphic to matrix rings over $D$-valuation rings. Our proof is somewhat different from the one given in [9].

Theorem 2.4. Let $R$ be a $(D)_{n}$-valuation ring with $D$ a division ring. Then there exists a $D$-valuation ring $T$ in $D$ and a regular element $d$ in $(D)_{n}$ with $d^{-1} R d=(T)_{n}$.

Proof. Let $e_{i j}$ be the matrix unit in $Q=(D)_{n}$ with 1 in the $(i-j)$-position and zero elsewhere. Consider $\sum_{i, j} R e_{i j}=L$ and $L$ is a cyclic left $R$-module in $Q$, using Theorem 2.3, and $L=R d$ for some $d$ in $Q$ follows. The element $d$ is regular in $Q$ since 1 is in $L$.

We show that $d^{-1} R d=d^{-1} L=\sum_{i, j} d^{-1} R e_{i j}$ contains all the matrix units $e_{k t}$.

The identity 1 is contained in $d^{-1} R d$, hence $1=\sum_{i, j} d^{-1} r_{i j} e_{i j}$ for some $r_{i j}$ in $R$ and $e_{k t}=1 e_{k t}=\sum_{i} d^{-1} r_{i k} e_{i t} \in d^{-1} R d$. This implies $d^{-1} R d=(T)_{n}$ for the subring $T=\left\{a_{11} \in D \mid\left(a_{i j}\right) \in d^{-1} R d\right\}$ of $D$.

It remains to prove that $T$ is a $D$-valuation ring. Since $(T)_{n} / J\left((T)_{n}\right) \cong$ $(T / J(T))_{n}$ and $R / J(R)$ is simple artinian it follows that $T / J(T)$ is simple artinian. To check the second condition let $q \in D \backslash T$ and $q e_{11} \in Q \backslash(T)_{n}$. Then $d\left(q e_{11}\right) d^{-1} \in Q \backslash R$ and $d q e_{11} d^{-1} r \in R \backslash J(R)$ for some $r$ in $R$. It follows that $q e_{11}\left(d^{-1} r d\right) \in(T)_{n} \backslash J\left((T)_{n}\right)$ and $q s_{1 i} \in T \backslash J(T)$ for at least one entry $s_{1 i} \in T$ in the first row of the matrix $d^{-1} r d \in(T)_{n}$. 
We later need the following results [9, Lemma 7].

Lemma 2.5. Let $R$ be a $Q$-valuation ring, $Q$ simple artinian. Then $O_{r}(J(R))=$ $O_{e}(J(R))=R$.

Here, $O_{r}(I)=\{q \in Q \mid I q \subseteq I\}$ is the right order of an additive subgroup $I$ of $Q$. The left order $O_{e}(I)$ is defined similarly.

For the rest of this section let $Q$ be a simple algebra, finite dimensional over its center $K$. We recall the following results:

Proposition 2.6 [9]. Let $R$ be a $Q$-valuation ring and $s R \supseteq R s$ or $R s \supseteq s R$ for some $s$ in $Q$. Then $s R^{\prime}=R^{\prime} s$ for any ring $R^{\prime}$ with $R \subseteq R^{\prime} \subseteq Q$. Further, $s^{n} R=k R$ for some natural number $n$ and some $k$ in $K$.

The next result shows that the assumption of the previous proposition holds for finitely generated $R$-ideals if $R$ is a maximal $Q$-valuation ring.

Proposition 2.7 [9]. Let $R$ be a $Q$-valuation ring and $T$ a finitely generated $R$-ideal. Then $T=s S=S s$ for some $s$ in $Q$ and $S=O_{r}(T)=O_{e}(T)$.

Here, an additive subgroup $I$ of $Q$ is called a right $R$-ideal if $I R=I, I$ contains a regular element and $a I \subseteq R$ for some regular element $a$ in $Q$. An $R$-ideal is a right and left ideal.

The next result [10, Theorem 1] describes the relationship between prime ideals in $R$ (not necessarily completely prime) and prime ideals in $V=R \cap K$. As in the commutative case, the overrings of $R$ can be described as localizations of $R$ on its prime ideals.

Theorem 2.8. Let $R$ be a $Q$-valuation ring for a simple algebra $Q$, finite dimensional over its center $K$. Then the following statements are true:

(i) $R \cap K=V$ is a valuation ring of $K$.

(ii) The mapping $\varphi(P)=P \cap K$ is an injective mapping from the set $\operatorname{spec}(R)$ of prime ideals of $R$ onto $\operatorname{spec}(V)$.

(iii) The mapping $\psi(S)=S \cap K$ from the set of rings $S$ with $R \subseteq S \subseteq Q$ is an injective mapping onto the set of rings between $V$ and $K$.

(iv) The set $C(P)$ of elements in $R$ which are regular mod $P$ is an Ore set in $R$ for $P$ in $\operatorname{spec}(R)$. The localization $R_{P}$ exists and $R_{P}=R V_{\varphi(P)}$.

(v) The mapping $\chi(P)=R_{P}$ is an injective mapping from $\operatorname{spec}(R)$ onto the set of rings between $R$ and $Q$.

Let $Q$ be a central simple algebra over the center $K$ with $[Q: K]=n$ and $V$ a valuation ring in $K$. We want to show that there exists a $Q$-valuation ring $R$ with $R \cap K=V$. This result was proved by Dubrovin in [10]; our proof is influenced by this proof, but differs in some essential points. 
Lemma 3.1. Let $R$ be a valuation ring in the simple artinian ring $Q$ and $q$ an element in $Q$ not in $R$. Then there exists $j$ in $J(R)$ with qj in $R \backslash J(R)$.

To prove this assume $q J(R) \cap R \subseteq J(R)$. If $q m$ is not in $R$ for some $m$ in $J(R)$ then $q m r \in R \backslash J(R)$ for some $r$ in $R$-a contradiction. We have $q J(R) \subseteq J(R)$ and $q \in O_{e}(J(R))$ - a contradiction to Lemma 2.5.

This result is used to show that certain subrings of a $Q$-valuation ring $R$ are again $Q$-valuation rings, which in turn will be used in an induction argument to prove the main result.

Lemma 3.2. Let $R$ be a $Q$-valuation ring in the simple artinian ring $Q$ and $S$ a subring of $R$ with $S \supseteq J(R)$. If $\bar{S}=S / J(R)$ is an $R / J(R)$-valuation ring then $S$ is a $Q$-valuation ring.

Proof. Let $\varphi$ be the canonical homomorphism from $S$ to $S / J(R)$ and denote $\varphi^{-1}(J(S / J(R))$ by $M$.

We have $S / M \cong \bar{S} / J(\bar{S})$ which is simple artinian since $\bar{S}$ is a valuation ring.

Next, let $q \in Q \backslash S$. If $q \in R \backslash S$ then there exists $s$ in $S$ with $\varphi(q) \varphi(s)=$ $\varphi(r)$ where $r$ is in $S \backslash M$ and $\varphi$ is extended to $R$ to denote the mapping modulo $J(R)$. The element $q s-r$ is contained in $J(R)$ and $q s \in S \backslash M$ follows. If $q \in Q \backslash R$, then by Lemma 3.1 there exists $j$ in $J(R)$ with $q_{0}=$ $q j \in R \backslash J(R)$. We can assume that $\bar{S}=S / J(R) \neq R / J(R)=\bar{R}=\left(D_{0}\right)_{m}$ for a division ring $D_{0}$. By Theorem 2.4 there exists a regular element $d$ in $\bar{R}$ with $d^{-1} \bar{S} d=\left(T_{0}\right)_{m}$ for a $D_{0}$-valuation ring $T_{0}$, and $d^{-1} \bar{M} d=\left(J\left(T_{0}\right)\right)_{m}$ follows. Since $d^{-1} \bar{q}_{0} d \neq 0$ in $\bar{R}=\left(D_{0}\right)_{m}$, there exists an element $r_{1}$ in $R$ with $d^{-1} \bar{q}_{0} d d^{-1} \bar{r}_{1} d=d^{-1} \bar{q}_{0} \bar{r}_{1} d \notin\left(J\left(T_{0}\right)\right)_{m}$ and hence $\bar{q}_{0} \bar{r}_{1} \in \bar{R} \backslash \bar{M}$. By the above argument there exists an element $s$ in $S$ with $q j r_{1} s \in S \backslash M$ and $j r_{1} s \in S$; it follows that $S$ is a $Q$-valuation ring.

We need one more technical lemma.

Lemma 3.3. Let $Q$ be a finite dimensional simple algebra over its center $K$ and $V$ a valuation ring of $K$. Assume that $R$ is a $Q$-valuation ring with $R \cap K=V$. Then there exists for any $q$ in $Q$ a nonzero element $v$ in $V$ with $q v$ in $R$.

Proof. We assume first that $Q$ is a division ring and $q \notin R$. Then there exists $r(\neq 0)$ in $R$ with $q r \in R \backslash J(R)$. Let $f(x) \in V[x]$ be a polynomial of minimal degree in $V[x]$ with $f(r)=0, f(x)=a_{n} x^{n}+\cdots+a_{1} x+a_{0}$, and $a_{0} \neq 0$. We have $q a_{0}=-q r\left(a_{n} r^{n-1}+\cdots+a_{1}\right) \in R$.

If now $Q=(D)_{n}$, then we can assume $R=(T)_{n}, T$ a $D$-valuation ring, $q=\left(q_{i j}\right), q_{i j} \in D$ (Theorem 2.4). Applying the first part of the proof there exists $v$ in $V, v \neq 0$, with $q_{i j} v \in T$ for all $i, j$ and $q v \in R$ follows.

The next result shows that rank 1 valuation rings in $K$ can be extended.

Lemma 3.4. Let $Q$ be a simple algebra finite dimensional over its center $K, V$ a maximal (i.e. rank 1) valuation ring of $K$. Then there exists a $Q$-valuation ring $R$ with $R \cap K=V$. 
Proof. Let $\widetilde{K}$ be the completion of $K$ with respect to the valuation defined by $V$ (i.e. with respect to the topology defined by the ideals of $V$ ) and denote with $\widetilde{V}$ the corresponding completion of $V$. Then $C=Q \otimes_{K} \widetilde{K}$ is a finite dimensional central simple algebra over $\widetilde{K}$ [24] and we identify $V, K, \widetilde{K}$ and $Q$ with their canonical images in $C=Q \widetilde{K}$. There is an isomorphism $\varphi$ from $C$ onto $(D)_{n}$ for some division ring $D$ and $\varphi(\widetilde{K})$ is a subfield of $D$.

By a well-known result $[26$, p. 53] there exists a total valuation subring $T$ of $D$ with $T \cap \varphi(\widetilde{K})=\varphi(\widetilde{V})$ and $S^{\prime}=(T)_{n}$ is a $(D)_{n}$-valuation ring with $S^{\prime} \cap \varphi(\tilde{K})=\varphi(\widetilde{V})$. We denote $\varphi^{-1}\left(S^{\prime}\right)$ by $S$ and $S$ is a $C$-valuation ring with $S \cap \widetilde{K}=\widetilde{V}$.

We denote $S \cap Q$ by $R$ and $J(S) \cap Q$ by $M$ and want to show that $R$ is a $Q$-valuation ring with $R \cap Q=V$. We claim first that $R+J(S)=S$.

The left-hand side is clearly contained in $S$. Let $s \in S$ and

$$
s=q_{1} k_{1}+\cdots+q_{n} k_{n}, \quad k_{i} \in \widetilde{K}, q_{i} \in Q .
$$

By Lemma 3.3 there exists $0 \neq a$ in $\widetilde{V}$ with $q_{i} a \in J(S)$. Since $K$ is dense in $\widetilde{K}$, there exist elements $k_{i}^{\prime}$ in $K$ with $\tilde{v}\left(k_{i}-k_{i}^{\prime}\right) \leq \tilde{v}(a)$ where $\tilde{v}$ is the valuation corresponding to $\widetilde{V}$. We obtain

$$
s=\sum q_{i} k_{i}^{\prime}+\sum q_{i}\left(k_{i}-k_{i}^{\prime}\right) \text { where } \sum q_{i}\left(k_{i}-k_{i}^{\prime}\right) \text { is in } J(S),
$$

$\sum q_{i} k_{i}^{\prime}$ is in $Q$ and also in $S$ and hence in $R$, which proves the claim.

We apply this and obtain

$$
\frac{R}{M}=\frac{Q \cap S}{Q \cap J(S)} \cong \frac{(Q \cap S)+J(S)}{J(S)}=\frac{S}{J(S)}
$$

is simple artinian.

Let $q$ be in $Q \backslash R$, then $q \in C \backslash S$ and $s$ exists in $S$ with $q s \in S \backslash J(S)$. As before: $s=\sum_{1}^{n} q_{i} k_{i}, q_{i} \in Q, k_{i} \in \widetilde{K}$. By Lemma 3.3 there exists an element $0 \neq a$ in $\widetilde{V}$ with $q q_{i} a, q_{i} a \in J(S)$ for all $i$. Further, there exist elements $k_{i}^{\prime}$ in $K$ with $\tilde{v}\left(k_{i}^{\prime}-k_{i}\right) \leq \tilde{v}(a)$.

We have $s=\sum q_{i} k_{i}^{\prime}+\sum q_{i}\left(k_{i}-k_{i}^{\prime}\right)$ where the first sum is an element in $Q \cap S$ and the second an element in $J(S)$. Hence, $q \sum q_{i} k_{i}^{\prime}=q s-\sum q q_{i}\left(k_{i}-k_{i}^{\prime}\right)$ is in $(Q \cap S) \backslash(Q \cap J(S))$ since the last sum is in $J(S)$ and $q s$ is in $S \backslash J(S)$. We conclude that $R=Q \cap S$ is a $Q$-valuation ring with $R \cap K=V$.

This last lemma can be applied immediately to prove the next result in case the valuation ring $V$ in $K$ has a minimal prime ideal $P$.

Lemma 3.5. Let $Q$ be a finite dimensional central simple algebra over $K, V$ $(\neq K)$ a valuation ring of $K$. Then there exists a $Q$-valuation ring $R$ with $R \neq Q$ and $R \cap Q \supseteq V$.

Proof. If $V$ contains a minimal prime ideal $P$ one localizes $V$ at $P$, applies Lemma 3.4 and obtains the result. We now assume that $V$ does not contain a minimal prime ideal. 
Let $\left\{m_{1}, m_{2}, \ldots, m_{n}\right\}$ be any $K$-basis of $Q$ with $m_{1}=1$. We have $m_{i} m_{j}=\sum a_{i j t} m_{t}, a_{i j t} \in K$, and an element $0 \neq c$ exists in $K$ with $c a_{i j t} \in V$ for all $i, j, t$. Consider the $K$-basis $\left\{1, a_{2}, \ldots, a_{n}\right\}$ with $a_{i}=c m_{i}, i=$ $2, \ldots, n$.

We now use the following result of Azumaya [2, Theorem 12]: A $K$-algebra $A$ with basis $\left\{a_{1}, \ldots, a_{n}\right\}$ over $K$ is centrally simple over $K$ if and only if the $n \times n$ matrix $N=\left(c_{i j}\right), c_{i j}=a_{j} a_{i}$ has an inverse in $(A)_{n}$.

If we apply this result to $Q$ and the basis $\left\{1, a_{2}, \ldots, a_{n}\right\}$ as above we obtain an inverse $N^{-1}=\left(b_{i j}\right)$ of $N$ in $(Q)_{n}$. We have $b_{i j}=\sum_{t} \beta_{i j t} a_{t}$ with $\beta_{i j t}$ in $K, a_{1}=1$. Since $V$ has no minimal prime ideal there exists a localization $V^{\prime} \neq K$ of $V$ with $\beta_{i j t} \in V^{\prime}$ for all $i, j, t$. We denote with $M^{\prime}$ the maximal ideal $J\left(V^{\prime}\right)$ of $V^{\prime}$. Then $R=\sum_{i=1}^{n} V^{\prime} a_{i}$ is a subring of $Q$ and $M=\sum M^{\prime} a_{i}$ is an ideal of $R$. It follows that $\left\{\bar{a}_{1}, \ldots, \bar{a}_{n}\right\}$ is a $V^{\prime} / M^{\prime}$-basis for $R / M$ and the matrix $\bar{N}=\left(\overline{c_{i j}}\right)$ has an inverse in $(R / M)_{n}$-where the bar denotes the mapping modulo $M$. By the above-mentioned result of Azumaya it follows that $R / M$ is simple artinian. Finally, for each $q$ in $Q \backslash R$ there exists an $s$ in $V^{\prime}$ with $q s \in R \backslash M-R$ is a $Q$-valuation ring with $R \cap Q \supseteq V$ and $R \neq Q$.

Lemma 3.6. Let $D$ be a division ring with center $K$ and $[D: K]=n$. Let $V$ be a valuation ring in $K, B_{1}$ and $B_{2}$ two distinct total valuation rings in $D$ with $B_{1} \cap K=V=B_{2} \cap K$. Then

$$
\left[\frac{B_{1}}{M_{1}}: \frac{V}{M}\right]+\left[\frac{B_{2}}{M_{2}}: \frac{V}{M}\right] \leq n \text { where } J\left(B_{i}\right)=M_{i}, J(V)=M .
$$

To prove this, observe that $B_{i} \nsubseteq B_{j}$ using Theorem 2.8 . We will give a direct proof without applying this result. If $B_{1} \supset B_{2}$ then $B_{1} \supset B_{2} \supset M_{2} \supset M_{1}$ and $M_{2}, M_{1}$ are completely prime ideals in $B_{2}$.

Let $m_{2} \in M_{2} \backslash M_{1}$ and elements $v_{i}$ exist in $V$, not all in $M$, with

$$
v_{k} m_{2}^{k}+\cdots+v_{1} m_{2}+v_{0}=0, \quad v_{0} \neq 0 .
$$

It follows that $v_{0}=-m_{2}\left(v_{1}+v_{2} m_{2}+\cdots+v_{k} m_{2}^{k-1}\right) \in M_{2} \cap K=M$, hence $v_{0} \in M_{1}$ and $v_{1}+v_{2} m_{2}+\cdots+v_{k} m_{k}^{k-1} \in M_{1} \subset M_{2}$. As before, $v_{1} \in M_{2} \cap K=$ $M \subseteq M_{1}$. Iterating this process leads to the contradiction $v_{i} \in M_{2}$ for all $i$.

This shows that $B_{i} \nsubseteq B_{j}$. Therefore, there exists $x_{1}^{\prime} \in B_{1}, x_{1}^{\prime} \notin B_{2}$. We can assume that $x_{1}^{\prime}$ is not in $M_{1}$, otherwise replace $x_{1}^{\prime}$ by $1+x_{1}^{\prime}$. The element $x_{1}=x_{1}^{\prime-1}$ is then contained in $B_{1} \backslash M_{1}$ and in $M_{2}$. Similarly, there exists an element $x_{2} \in\left(B_{2} \backslash M_{2}\right) \cap M_{1}$.

Let $a_{1}, \ldots, a_{r}$ be in $B_{1}$ such that $\left\{a_{i}+M_{1} \mid i=1, \ldots, r\right\}$ is a basis of $B_{1} / M_{1}$ over $V / M$. We can assume that $a_{1} B_{2} \supseteq a_{i} B_{2}, i=1, \ldots, r$ and after replacing $a_{i}$ by $x_{1} a_{1}^{-1} a_{i}$ we can assume that the $a_{i}$ are all in $M_{2}$.

Similarly, we can find a basis $\left\{b_{j}+M_{2} \mid j=1, \ldots, s\right\}$ for $B_{2} / M_{2}$ over $V / M$ with $b_{j} \in B_{2} \cap M_{1}$. 
If the set $\left\{a_{1}, \ldots, a_{r}, b_{1}, \ldots, b_{s}\right\}$ is linearly dependent then there exists an equation $\sum w_{i} a_{i}+\sum w_{j}^{\prime} b_{j}=0, w_{i}, w_{j}^{\prime} \in V$, not all in $M$. This leads to a contradiction.

Lemma 3.7. Let $Q$ be a central simple algebra over $K$ with $[Q: K]=n$ and $R$ a $Q$-valuation ring with $R \cap K=V, V$ a valuation ring of $K$. Then $[R / J(R): V / J(V)] \leq n$.

It is only necessary to observe that $\left\{r_{i} \mid i=1, \ldots, t\right\}, r_{i}$ in $R$, is linearly independent over $K$ if $\left\{r_{i}+J(R) \mid i=1, \ldots, t\right\}$ is a basis for $R / J(R)$ over $V / J(V)$.

We can now prove the main result of this section.

Theorem 3.8. Let $Q$ be a simple algebra with center $K$ and $[Q: K]=n$ and $V$ a valuation ring in $K$. Then there exists a $Q$-valuation ring $R$ with $R \cap K=V$.

Proof by induction on $n$, where the case $n=1$ is trivial. In the general case let $\mathscr{R}^{\prime}$ be the set of all $Q$-valuation rings $R$ in $Q$ with $Q \neq R$ and $R \cap K \supseteq V$. (We can assume that $V \neq K$.)

The set $\mathscr{R}^{\prime}$ is not empty by Lemma 3.5. We assume first that there exists a ring $R$ in $\mathscr{R}^{\prime}$ with $R / J(R) \cong\left(D_{0}\right)_{m}$ and $D_{0}$ a division ring, $m>1$. Then we can assume $R / J(R)=\left(D_{0}\right)_{m}$ and obtain, using Lemma 3.7,

$$
\left[D_{0}: \frac{R \cap K}{J(R) \cap K}\right]<\left[\frac{R}{J(R)}: \frac{R \cap K}{J(R) \cap K}\right] \leq n .
$$

We have $R \cap K \supseteq V \supset J(V) \supseteq J(R) \cap K=J(R \cap K)$. By induction there exists a $D_{0}$-valuation ring $T_{0}$ with

$$
T_{0} \cap\left(\frac{R \cap K}{J(R) \cap K}\right)=\frac{V}{J(R) \cap K} \quad \text { and } \quad\left(T_{0}\right)_{m}=\frac{S}{J(R)}
$$

is an extension of $V /(J(R) \cap K)$ in $R / J(R)$ for a subring $S$ of $R$ with $S \supseteq$ $J(R)$. By Lemma 3.2 it follows that $S$ is a $Q$-valuation ring and $S \cap K=V$.

We must now consider the case where $J(R)$ is completely prime in $R$ for every $R$ in $\mathscr{R}^{\prime}$. It follows (Lemma 2.2, Theorem 2.4) that $Q=D$ is a division ring and $R$ is a total valuation ring in $D$ for every $R$ in $\mathscr{R}^{\prime}$.

If there exists an $R$ in $\mathscr{R}^{\prime}$ with

$$
\left[\frac{R}{J(R)}: \frac{R \cap K}{J(R) \cap K}\right]<n
$$

we can use induction to extend $V /(J(R) \cap K)$ to $R / J(R)$ and obtain with Lemma 3.2 a $D$-valuation ring $S$ with $S \cap K=V$.

We are left with the case that all $R$ in $\mathscr{R}^{\prime}$ are total valuation rings and

$$
\left[\frac{R}{J(R)}: \frac{R \cap K}{J(R) \cap K}\right]=n \quad \text { for all } R \text { in } \mathscr{R}^{\prime} .
$$

We will prove below that then $R_{1} \subseteq R_{2}$ or $R_{2} \subseteq R_{1}$ for any $R_{1}, R_{2} \in \mathscr{R}^{\prime}$. 
Assuming this fact let $R_{0}$ be the intersection of all $R_{i}$ in $\mathscr{R}^{\prime}$ and $R_{0}$ is the minimal element in $\mathscr{R}^{\prime}$. If $R_{0} \cap K \supset V$, then

$$
\frac{R_{0}}{J\left(R_{0}\right)} \supset \frac{R_{0} \cap K}{J\left(R_{0}\right) \cap K} \supset \frac{V}{J\left(R_{0}\right) \cap K}
$$

and by Lemma 3.5 an $R_{0} / J\left(R_{0}\right)$ valuation ring $S / J\left(R_{0}\right) \neq R / J\left(R_{0}\right)$ exists with

$$
\frac{S}{J\left(R_{0}\right)} \cap \frac{R_{0} \cap K}{J\left(R_{0}\right) \cap K} \supseteq \frac{V}{J\left(R_{0}\right) \cap K}
$$

and $R_{0} \supset S$ and $S \cap K \supseteq V$ is a contradiction.

To prove the above claim let $R_{1}, R_{2} \in \mathscr{R}^{\prime}$ and we can assume that $R_{1} \cap K \subseteq$ $R_{2} \cap K$ (these rings are overrings of $V$ ). We will show that $R_{1} \subseteq R_{2}$.

The ring $\left(R_{2} \cap K\right) R_{1}$ is an overring of $R_{1}$. We show that $\left(R_{2} \cap K\right) R_{1} \cap K=$ $R_{2} \cap K$. Let $s_{1} r_{1}+s_{2} r_{2}+\cdots+s_{t} r_{t}=k \in K$ with $s_{i} \in R_{2} \cap K, r_{i} \in R_{1}$ for all $i$. If $k \notin R_{2} \cap K$ then $k^{-1}$ and hence $k^{-1} s_{i} \in J\left(R_{2} \cap K\right) \subseteq J\left(R_{1} \cap K\right)$, a contradiction, since then $\sum k^{-1} s_{i} r_{i}=1 \in J(R)$. We can apply Lemma 3.6 to $B_{1}=\left(R_{2} \cap K\right) R_{1}$ and $B_{2}=R_{2}$ and obtain $B_{1}=B_{2}$ and $R_{1} \subseteq R_{2}$.

\section{4}

In this section let $D$ be a division ring finite dimensional over its center $K$ and $V$ a valuation ring in $K$. Let $\mathscr{R}$ be the set of $D$-valuation rings $R$ with $R \cap K=V$ and we know by Theorem 3.8 that $|\mathscr{R}| \geq 1$. We show that $|\mathscr{R}|=1$ if $\mathscr{R}$ contains an invariant total valuation ring and that every element in $\mathscr{R}$ is total if one of them is.

Lemma 4.1. Let $D$ be a division ring finite dimensional over its center $K$. Let $V$ be a valuation ring of $K$. Assume that the set $\mathscr{R}$ of extensions of $V$ in $D$ contains an invariant total valuation ring $B$, then $\mathscr{R}=\{B\}$.

For a proof assume that $R$ is a $D$-valuation ring with $R \cap K=V$. We want to show that $R=B$ and claim first that $J(R) \subseteq J(B)$. To prove this let $0 \neq m$ be an element in $J(R)$ and let $f(x)=x^{n}+a_{n-1} x^{n-1}+\cdots+a_{1} x+a_{0}$ be the minimal polynomial of $m$ over $K$. Assume that $m^{-1}$ is in $B$ hence integral over $V$ [5, Theorem 3]. This implies that $x^{n}+a_{0}^{-1} a_{1} x^{n-1}+\cdots+a_{0}^{-1} a_{n-1} x+a_{0}^{-1}=$ $g(x)$ is the minimal polynomial of $m^{-1}$ over $K$ and $a_{0}^{-1} a_{i} \in V$ follows for $i=0, \ldots, n$ with $a_{n}=1$. Using $f(m)=0$ we obtain

$$
a_{0}^{-1} m^{n}+a_{0}^{-1} a_{n-1} m^{n-1}+\cdots+a_{0}^{-1} a_{1} m=-1 \in J(R), \quad \text { a contradiction. }
$$

Hence, $m^{-1} \notin B$ which implies $m \in J(B)$ and $J(R) \subseteq J(B)$ follows. Next, assume that $B \neq R$. Then $B$ and $R$ are two distinct $D$-valuation rings which are extensions of $V$ and by Theorem 2.8 it follows that $R \nsubseteq B$. Let $r \in R$, $r \notin B$. If $[D: K]=n$ then $1, r, r^{2}, \ldots, r^{n}$ are linearly dependent over $K$ and elements $k_{i}$ exist in $K$, not all zero, with $\sum_{0}^{n} k_{i} r^{i}=0$. Hence, there exist 
$j>i$ with $k_{i} r^{i} \neq k_{j} r^{j}$ and $k_{i} r^{i} B=k_{j} r^{j} B$ and $r^{j-i} B=k_{j}^{-1} k_{i} B$ follows, i.e. $r^{t} B=k B$ for $t=j-i$ and an element $k$ in $K$.

Since $r \notin B$, we have $r^{t} \notin B, k \notin B$ and $k^{-1} \in J(B) \cap K=J(R) \cap$ $K=J(V)$. The element $k^{-1} r^{t}$ is a unit in $B$ and $k^{-1} r^{t} \notin J(B)$. But $k^{-1} r^{t} \in J(R) \subseteq J(B)$, a contradiction that proves $R=B$.

Corollary 4.2. Let $D$ be a finite dimensional division algebra over its center $K$. Assume that $K$ is complete with respect to a rank 1 valuation with corresponding valuation ring $V$. Then there exists exactly one $D$-valuation ring $R$ with $R \cap$ $K=V$.

Proof. It follows from [26, p. 53] that there exists an invariant total valuation ring $B$ of $D$ with $B \cap K=V$ and the statement follows from Lemma 4.1.

The next result deals with the case that the set $\mathscr{R}$ of extensions contains a total valuation ring.

Theorem 4.3. Let $D$ be a division ring finite dimensional over its center $K$ and $V$ a valuation ring in $K$. Assume that the set $\mathscr{R}$ of extensions of $V$ in $D$ contains a total valuation ring $B$. Then every extension $R$ in $\mathscr{R}$ is a total valuation ring.

Proof. We use induction on $n=[D: K]$ and the case $n=1$ is trivial. In the general case assume first that $B$ is the only total valuation ring extending $V$. Then $B$ is also invariant and the result follows from Lemma 4.1.

If we assume that $B$ is not the only total valuation ring in $\mathscr{R}$, there exists [5, Lemma 4] an invariant total valuation ring $T$ in $D, T \neq D$, that contains all total valuation rings in $\mathscr{R}$. Let $R$ be an arbitrary element in $\mathscr{R}$. It follows from Theorem 2.8 that there exists in $R$ a prime ideal $P$ with $P \cap V=J(T) \cap V$ and a $D$-valuation ring $R_{1} \supseteq R$ with $J\left(R_{1}\right)=P$ and $R_{1} \cap K=T \cap K$. Since $T$ is invariant, Lemma 4.1 implies $T=R_{1}$ and $P=J(T)$. The division ring $T / J(T)$ contains $R / J(T)$ and $B / J(T)$ as $T / J(T)$-valuation rings which are extensions of $V /(J(T) \cap K)$.

By Lemma 5 in [5] and our assumption it follows that $(T \cap K) /(J(T) \cap K)$ is properly contained in the center $Z$ of $T / J(T)$ and $[T / J(T): Z]<n$.

The intersection $R / J(T) \cap Z$ is a valuation ring of the commutative field $Z$. Again by Lemma 5 in [5] it follows that the separable closure $S$ of $(T \cap$ $K) /(J(T) \cap K)$ in $Z$ is a Galois extension such that the $(T \cap K) /(J(T) \cap K)$ automorphisms of $S$ are induced by inner automorphisms of $D$.

Hence, there exists an element $0 \neq d$ in $D$ with $\left(d B d^{-1} / J(T)\right) \cap Z=$ $(R / J(T)) \cap Z$ and $d B d^{-1} / J(T)$ is an extension of $R / J(T) \cap Z$ which is a total valuation ring in $T / J(T)$. By induction $R / J(T)$ is a total valuation ring in $T / J(T)$, the ring $T$ is total and hence $R$ is total which proves the theorem. 
It is shown in this section that the elements in the set $\mathscr{R}$ of $Q$-valuation rings $R$ with $R \cap K=V$ are conjugate in $Q$ if $Q$ is a simple algebra finite dimensional over its center $K$ and $V$ is a valuation ring of $K$ of finite rank.

The rank 1 case is proved by using the completion of $Q$ with respect to the topology given by the finitely generated ideals in a $Q$-valuation ring $R$. A norm can be defined on $Q$ that extends the valuation of $K$ given by $V$.

Let $Q$ be a central simple algebra finite dimensional over its center $K$. Let $R$ be a maximal $Q$-valuation ring and it follows from Theorem 2.8 that this is the case if and only if $(0)$ and $J(R)$ are the only prime ideals in $R$ if and only if $R \cap K=V$ is a maximal (i.e. rank 1) valuation ring in $K$. Denote with $v$ the corresponding valuation of $K$ whose value group is isomorphic to a subgroup of $\left(\mathbb{R}^{+}, \circ\right)$ the ordered group of positive real numbers under multiplication.

If $I$ is a finitely generated $R$-ideal in $Q$, then $O_{e}(I)=R=O_{r}(I)$ and $I=r R=R r$ for some regular element in $Q$ (Propositions 2.6 and 2.7). It follows that the set $G=\{r R \mid r$ regular in $Q$ and $r R=R r\}$ is an ordered group.

We show that $G$ can be considered as a subgroup of $\left(\mathbb{R}^{+}, 0\right)$ by checking the archimedian property for $G$ : Let $s R, s^{\prime} R \in G, s R, s^{\prime} R \subseteq J(R)$. By Proposition 2.6 there exist natural numbers $n$ and $n^{\prime}$ and $k, k^{\prime}$ in $J(V)$ with $s^{n} R=k R, s^{\prime n^{\prime}} R=k^{\prime} R$. But $V$ is maximal and there exists $m$ with $k^{m} \in k^{\prime} V \subseteq k^{\prime} R$ and $s^{n m} R=k^{m} R \subseteq k^{\prime} R=s^{n^{\prime}} R \subseteq s^{\prime} R$ follows.

The mapping $N$ from $Q$ to $\widehat{G}=G \cup\{0\}$ defined by $N(q)=R q R$ considered as element of $\widehat{G}$ has the following properties:

(i) $N(q)=0$ if and only if $q=0$.

(ii) $N(k q)=v(k) N(q)$ for $0 \neq k$ in $K, q$ in $Q$.

(iii) $N\left(q+q^{\prime}\right) \leq \max \left\{N(q), N\left(q^{\prime}\right)\right\}$.

The mapping $N$ defines a norm on $Q$, which is a topological vectorspace with respect to $N$ and $v$. In addition, the following property holds:

(iv) $N\left(q q^{\prime}\right) \leq N(q) N\left(q^{\prime}\right)$ for $q$ and $q^{\prime}$ in $Q$ and $Q$ is a topological ring.

Let $\widetilde{Q}$ be the completion of $Q$ with respect to $N$ (i.e. $\widetilde{Q}=\widehat{Q} / A$ where $\widehat{Q}$ is the ring of all Cauchy sequences and $A$ is the two-sided ideal of all null sequences in $\widehat{Q}$ ). We also say that $\widetilde{Q}$ is the completion of $Q$ with respect to $R$. Let $\widetilde{K}$ be the completion of $K$ with respect to $v$ and we consider $\widetilde{K}$ as a subring of the center of $\widetilde{Q}$.

Let $0 \neq q \in \widetilde{Q}, q=\left(a_{i}\right)+A,\left(a_{i}\right) \notin A$. Then there exists a natural number $n_{0}$ with

$$
N\left(a_{n}\right)=N\left(a_{0}\right)>0 \text { for all } n \geq n_{0} .
$$

We define $\tilde{N}(q)=N\left(a_{0}\right), \tilde{N}(0)=0$ and $\tilde{N}$ defines a norm on $\widetilde{Q}$ considered as vectorspace over $\widetilde{K}$, in fact $\widetilde{N}$ satisfies (i)-(iv) for $\widetilde{Q}$ and $\widetilde{K}$. The restriction of $\widetilde{N}$ to $\widetilde{K}$ is a valuation $\tilde{v}$ that extends $v$ and $\widetilde{K}$ is complete with respect to $\tilde{v} . \quad Q$ and $\widetilde{K}$ can be considered as subrings of $\widetilde{Q}$ and the mapping 
$\varphi\left(\sum\left(g_{i} \otimes k_{i}\right)\right)=\sum q_{i} k_{i}$ defines an $K$-algebra homomorphism from $Q \otimes_{K} \widetilde{K}$ to $\widetilde{Q}$ and $\varphi$ is injective since $Q \otimes_{K} \widetilde{K}$ is a simple $\widetilde{K}$-algebra. The image $Q \widetilde{K}$ of $\varphi$ is a finite dimensional vector space over the complete field $\widetilde{K}$ and $\widetilde{N}$ induces a norm on $Q \widetilde{K}$. Every element $q$ in $\widetilde{Q}$ can be considered as the limit of a sequence $\left(a_{i}\right), a_{i}$ in $Q$. But $\left(a_{i}\right)$ is also a Cauchy sequence in the complete $\widetilde{K}$-vectorspace $Q \widetilde{K}$ and hence has a limit in $Q \widetilde{K}$. This implies $q$ in $Q \widetilde{K}$ and shows that $Q \otimes_{K} \widetilde{K}$ and $\widetilde{Q}$ are isomorphic; $\widetilde{Q}$ is a central simple algebra over $\widetilde{K}$. The set $\widetilde{R}=\{q \in \widetilde{Q} \mid \widetilde{N}(q) \leq \widetilde{N}(1)\}$ is a subring of $\widetilde{Q}$ and $\widetilde{M}=\{q \in \widetilde{Q} \mid \tilde{N}(q)<\widetilde{N}(1)\}$ is an ideal of $\widetilde{R}$. Since $Q$ is dense in $\widetilde{Q}$, there exists for every $q$ in $\widetilde{R}$ an element $r$ in $R$ with $\widetilde{N}(q-r)<\widetilde{N}(1)$ and $\widetilde{R} / \widetilde{M} \cong R / J(R)$ follows, i.e. $\widetilde{R} / \widetilde{M}$ is simple artinian.

Finally, for each $q \in \widetilde{Q} \backslash \widetilde{R}$ there exist $r, r^{\prime}$ in $\widetilde{R}$ with $q r^{\prime}, r q \in \widetilde{R} \backslash \widetilde{M}$ since $R$ is a $Q$-valuation ring and $Q$ is dense in $\widetilde{Q}$.

We summarize the above results.

Proposition 5.1. Let $Q$ be a simple algebra, finite dimensional over its center $K$ and $R$ be a maximal $Q$-valuation ring with $R \cap K=V$. Then $V$ is a rank 1 valuation ring of $K$. If $\widetilde{K}$ is the completion of $K$ with respect to $V$, then $Q \otimes_{K} \widetilde{K}$ is the completion $\widetilde{Q}$ of $Q$ with respect to $R$ and contains a $\widetilde{Q}$-valuation ring $\widetilde{R}$ with $\widetilde{R} \cap Q=R$ and $\widetilde{R} \cap \widetilde{K}=\widetilde{V}$, the completion of $V$.

We show in the next result that the extensions of a rank-1 valuation ring $V$ in the center $K$ of a simple ring $Q,[Q: K]<\infty$, are conjugate in $Q$.

Theorem 5.2. Let $Q$ be a simple algebra finite dimensional over its center $K$ and $V$ a rank-1 valuation ring of $K$. Then any two $Q$-valuation rings $R$ and $R^{\prime}$ with $R \cap K=V=R^{\prime} \cap K$ are conjugate in $Q$.

Proof. Let $\widetilde{Q} \supseteq \widetilde{R}$ and $\widetilde{Q}^{\prime} \supseteq \widetilde{R}^{\prime}$ be the completions of $Q$ with respect to $R$ and $R^{\prime}$ respectively. By Proposition 5.1 we have $\widetilde{Q} \cong Q \otimes_{K} \widetilde{K} \cong \widetilde{Q}^{\prime}$ and can assume the following: $\widetilde{Q}=Q \widetilde{K}$ contains two $\widetilde{Q}$-valuation rings $\widetilde{R}$ and $\widetilde{R}^{\prime}$ with

$$
\widetilde{R} \cap Q=R, \quad \widetilde{R}^{\prime} \cap Q=R^{\prime}, \quad \widetilde{R} \cap \widetilde{K}=\widetilde{R}^{\prime} \cap \widetilde{K}=\widetilde{V} .
$$

Further, $Q \widetilde{K} \cong(L)_{n}$ for some division ring $L$ and we can assume $Q \widetilde{K}=(L)_{n}$ with $L$ contained in $Q \widetilde{K}$ and finite dimensional over its center $\widetilde{K}$.

By Theorem 2.4 there exist regular elements $u, v \in Q \widetilde{K}$ with $u \widetilde{R} u^{-1}=(S)_{n}$, $v \widetilde{R}^{\prime} v^{-1}=\left(S^{\prime}\right)_{n}$ where $S, S^{\prime}$ are $L$-valuation rings. Then $S \cap \widetilde{K}=u \widetilde{R} u^{-1} \cap \widetilde{K}=$ $\widetilde{R} \cap \widetilde{K}=\widetilde{R}^{\prime} \cap \widetilde{K}=v \widetilde{R}^{\prime} v^{-1} \cap \widetilde{K}=S^{\prime} \cap \widetilde{K}$ and $S=S^{\prime}$ by Corollary 4.2 which implies $u \widetilde{R} u^{-1}=v \widetilde{R}^{\prime} v^{-1}$ and $d \widetilde{R} d^{-1}=\widetilde{R}^{\prime}$ for a regular element $d$ in $Q \widetilde{K}$.

Let $\left\{q_{1}, \ldots, q_{n}\right\}$ be a $K$-basis of $Q$ and $N$ the norm on $Q$ induced by $R$.

$$
d=k_{1} q_{1}+\cdots+k_{n} q_{n} \text { with } k_{i} \in \widetilde{K}, q_{i} \in Q .
$$

Since $K$ is dense in $\widetilde{K}$ there exist elements $k_{j}^{\prime}$ in $K$ with

$$
\tilde{v}\left(k_{j}-k_{j}^{\prime}\right)<N\left(d^{-1}\right)^{-1} N\left(q_{j}\right)^{-1} \text { for all } j .
$$


We have $d=\sum q_{i} k_{i}^{\prime}+\sum q_{i}\left(k_{i}-k_{i}^{\prime}\right)$ and

$$
\begin{aligned}
N\left(d^{-1}\left(\sum q_{i}\left(k_{i}-k_{i}^{\prime}\right)\right)\right) & \leq N\left(d^{-1}\right) N\left(\sum q_{i}\left(k_{i}-k_{i}^{\prime}\right)\right) \\
& \leq N\left(d^{-1}\right) \max \left\{N\left(q_{j}\right) \tilde{v}\left(k_{j}-k_{j}^{\prime}\right)\right\} \\
& <N\left(d^{-1}\right) \max \left\{N\left(q_{j}\right) N\left(d^{-1}\right)^{-1} N\left(q_{j}\right)^{-1}\right\}=1 .
\end{aligned}
$$

We have

$$
q=\sum q_{i} k_{i}^{\prime}=d\left(1-d^{-1}\left(\sum q_{i}\left(k_{i}-k_{i}^{\prime}\right)\right)\right) \in Q
$$

and

$$
w=1-d^{-1}\left(\sum q_{i}\left(k_{i}-k_{i}^{\prime}\right)\right)
$$

is a unit in $\widetilde{R}$. Hence, $d \widetilde{R} d^{-1}=d w \widetilde{R} w^{-1} d^{-1}=q \widetilde{R} q^{-1}=\widetilde{R}^{\prime}$ for $q$ in $Q$. Therefore, $q R q^{-1}=q(\widetilde{R} \cap Q) q^{-1}=\widetilde{R}^{\prime} \cap Q=R^{\prime}$ which proves Theorem 5.2.

Before we can prove that the elements in $\mathscr{R}$ are conjugate in $Q$ in case $V$ has finite rank we need the following result:

Proposition 5.3. Let $Q$ be a simple algebra finite dimensional over its center $K$ and $V$ a rank 1 valuation ring in $K$. Let $R$ be a $Q$-valuation ring with $R \cap K=V$ and let $Z$ be the center of $R / J(R)$ with $S$ the separable closure of $V / J(V)$ in $Z$.

Then $S$ is a Galois extension of $V / J(V)$ and every $V / J(V)$-automorphism of $S$ is induced by an inner automorphism of $Q$ that maps $R$ onto $R$.

Proof. Let $\widetilde{K}$ be the completion of $K$ with respect to (the topology given by the ideals of) $V$ with $\widetilde{V}$ the completion of $V$ and $C=Q \widetilde{K} \cong Q \otimes_{K} \widetilde{K} \cong(D)_{n}$ for some division ring $D$. Let $\varphi$ be the isomorphism from $C$ to $(D)_{n}$ and $\varphi(\widetilde{K})$ is then the center of $(D)_{n}$ which can also be considered as the center of $D$.

Since $\widetilde{V}$ is complete of $\operatorname{rank} 1, \varphi(\widetilde{V})$ has a unique extension $B$ in $D$ (Corollary 4.2) and $(B)_{n}$ is a $(D)_{n}$-valuation ring with $(B)_{n} \cap \varphi(\widetilde{K})=\varphi(\widetilde{V})$.

We have

$$
J\left((B)_{n}\right)=(J(B))_{n} \text { and }(B)_{n} / J\left((B)_{n}\right) \cong(B / J(B))_{n} .
$$

Hence, if $A$ denotes the center of $B / J(B)$ then $A$ is isomorphic to the center of $(B)_{n} / J\left((B)_{n}\right)$.

Let $S^{\prime}$ be the separable closure of $\varphi(\tilde{V}) / J(\varphi(\tilde{V}))$ in $A$. Since $B$ is an invariant total valuation ring of the skew field $D$, it follows that $S^{\prime}$ is a Galois extension of $\varphi(\tilde{V}) / J(\varphi(\widetilde{V}))$ and every $\varphi(\widetilde{V}) / J(\varphi(\widetilde{V}))$-automorphism of $S^{\prime}$ is induced by an inner automorphism of $D$. ((2) in Lemma 5 in [5] remains true without the condition $|\mathscr{B}|>1$.)

If we denote with $S^{\prime \prime}$ the separable closure of $\varphi(\tilde{V}) / J(\varphi(\tilde{V}))$ in the center of $(B)_{n} / J\left((B)_{n}\right)$-which is isomorphic to $A$-then $S^{\prime \prime}$ is a Galois extension of $\varphi(\tilde{V}) / J(\varphi(\tilde{V}))$ and the $\varphi(\tilde{V}) / J(\varphi(\tilde{V}))$-automorphisms of $S^{\prime \prime}$ are induced by inner automorphisms of $(D)_{n}$ that map $(B)_{n}$ onto $(B)_{n}$. 
The preimage $R_{C}=\varphi^{-1}\left((B)_{n}\right)$ is a $C$-valuation ring that extends $\tilde{V}$. The separable closure $S_{C}$ of $\tilde{V} / J(\tilde{V})$ in the center of $R_{C} / J\left(R_{C}\right)$ is a Galois extension of $\widetilde{V} / J(\widetilde{V})$ and every $\widetilde{V} / J(\widetilde{V})$-automorphism of $S_{C}$ is induced by an inner automorphism of $C$ corresponding to an invertible element $d$ in $C$ with $d R_{C} d^{-1}=R_{C}$.

In the last part of the proof of Theorem 5.2 it was shown that there exist $q$ in $Q$ and $j$ in $J\left(R_{C}\right)$ with $q=d(1-j)$ and $q$ induces the same automorphism on $R_{C} / J\left(R_{C}\right)$ as $d$ does.

In the proof of Lemma 3.4 it is shown that $R_{C} \cap Q=R^{\prime}$ is a $Q$-valuation ring and $R^{\prime} \cap K=V$. From the remarks before Proposition 4.4 it follows that $R_{C} / J\left(R_{C}\right)$ and $R^{\prime} / J\left(R^{\prime}\right)$ as well as $\widetilde{V} / J(\widetilde{V})$ and $V / J(V)$ are isomorphic. The statement of Proposition 5.3 is therefore true for $R^{\prime}$ and hence for $R$ which is conjugate to $R^{\prime}$ by Theorem 5.2.

Theorem 5.4. Let $Q$ be a simple algebra finite dimensional over its center $K$ and $V$ a valuation ring in $K$ of finite rank. Then any two $Q$-valuation rings $R$ and $R^{\prime}$ with $R \cap K=R^{\prime} \cap K=V$ are conjugate in $Q$.

The theorem will be proved by induction on $n$, the rank of $V$, and the case $n=1$ is just Theorem 5.2.

Let $T$ and $T^{\prime}$ be maximal $Q$-valuation rings that contain $R$ and $R^{\prime}$ respectively (Theorem 2.8). Then $T \cap K=V_{P}=T^{\prime} \cap K$ where $P$ is the minimal prime ideal in $V$. Theorem 5.2 implies that $T$ and $T^{\prime}$ are conjugate in $Q$, say $q T^{\prime} q^{-1}=T$ for some invertible element $q$ in $Q$ and $q J\left(T^{\prime}\right) q^{-1}=J(T)$ follows.

The simple algebra $\bar{Q}=T / J(T)$, which is finite dimensional over its center $Z$, contains the $\bar{Q}$-valuation rings $R / J(T)=\bar{R}$ and $\overline{q R^{\prime} q^{-1}}=q R^{\prime} q^{-1} / J(T)$. Let $S$ be the separable closure of $\bar{K}=T \cap K /(J(T) \cap K)$ in $Z$.

$Z$ is a field and $\bar{R} \cap Z$ and $\overline{q R^{\prime} q^{-1}} \cap Z$ are two valuation rings in $Z$ with $(\bar{R} \cap Z) \cap \bar{K}=\left(\overline{q R^{\prime} q^{-1}} \cap Z\right) \cap \bar{K}$. Since $T$ is a maximal $Q$-valuation ring we can apply Proposition 5.3 and conclude that there exists a regular element $r$ in $Q$ with $r T r^{-1}=T$ such that $\varphi_{r}\left(\overline{q R^{\prime} q^{-1}} \cap Z\right)=\bar{R} \cap Z$ where $\varphi_{r}$ is the mapping induced on $T / J(T)$ by the inner automorphism of $Q$ corresponding to $r$. Hence, $\overline{r q R^{\prime} q^{-1} r^{-1}} \cap Z=\bar{R} \cap Z$.

By induction, applied to $\bar{Q}, \bar{R}$ and $\overline{r q R^{\prime} q^{-1} r^{-1}}$ there exists in $T \backslash J(T)$ an element $a$ such that $\bar{a}$ is invertible in $\bar{Q}$ and $\overline{a r q R^{\prime} q^{-1} r^{-1}} \bar{a}^{-1}=\bar{R}$. This implies that $a$ is invertible in $Q$ and $\operatorname{arqR}^{\prime} q^{-1} r^{-1} a^{-1}=R$.

\section{REFERENCES}

1. S. A. Amitsur, On central division algebras, Israel J. Math. 12 (1972), 408-420.

2. G. Azumaya, On maximally central algebras, Nagoya Math. J. 2 (1951), 119-150.

3. H. H. Brungs, Rings with a distributive lattice of right ideals, J. Algebra 40 (1976), 392-400. 
4. H. H. Brungs and G. Törner, Extensions of chain rings, Math. Z. 185 (1984), 93-104.

5. H. H. Brungs and J. Gräter, Valuation rings in finite dimensional division algebras, J. Algebra 120 (1989), 90-99.

6. P. M. Cohn, On the embedding of rings in skew fields, Proc. London Math. Soc. (3) 11 (1961), 511-530.

7. P. M. Cohn and M. Mahdavi-Hezavehi, Extensions of valuations on skew fields, Proc. Ring Theory Week, Antwerp 1980 (Ed., F. Van Oystaeyen), Lecture Notes in Math., vol. 825, Springer-Verlag, Berlin and New York, 1980, pp. 28-41.

8. P. M. Cohn, On extending valuations in division algebras, Studia Sci. Math. Hungar. 16 (1981), $65-70$.

9. N. I. Dubrovin, Noncommutative valuation rings, Trudy Moskov. Mat. Obshch. 45 (1982), 265-289 = Trans. Moscow Math. Soc. 45 (1984), 273-287.

10. __ Noncommutative valuation rings in simple finite-dimensional algebras over a field, Mat. Sb. 123(165) (1984) = Math.-USSR Sb. 51 (1985), 493-505.

11. O. Endler, Valuation theory, Springer-Verlag, Berlin and New York, 1972.

12. A. W. Goldie, The structure of noetherian rings, Lectures on Rings and Modules, Lecture Notes in Math., vol. 246, Springer-Verlag, Berlin and New York, 1972, pp. 213-321.

13. J. Gräter, Zur Theorie nicht kommutativer Prüferringe, Arch. Math. 41 (1983), 30-36.

14. __ Über Bewertungen endlich dimensionaler Divisionsalgebren, Results in Math. 7 (1984), 54-57.

15. B. Jacob and A. R. Wadsworth, A new construction of noncrossed product algebras, Trans. Amer. Math. Soc. 293 (1986), 693-721.

16. W. Klingenberg, Projektive und affine Ebenen mit Nachbarelementen, Math. Z. 60 (1954), 384-406.

17. W. Krull, Allgemeine Bewertungstheorie, J. Reine Angew. Math. 167 (1932), 160-196.

18. J. Kuzmanovich, Localizations of Dedekind prime rings, J. Algebra 21 (1972), 378-393.

19. A. I. Lichtman, PI-subrings and algebraic elements in enveloping algebras and their fields of fractions (to appear).

20. K. Mathiak, Bewertungen nicht kommutativer Körper, J. Algebra 48 (1977), 217-235.

21. __ Valuations of skew fields and projective Hjelmsley spaces, Lecture Notes in Math., vol. 1175, Springer-Verlag, Berlin and New York, 1986.

22. B. H. Neumann, On ordered division rings, Trans. Amer. Math. Soc. 66 (1949), 202-252.

23. B. L. Osofsky, Noncommutative rings whose cyclic modules have cyclic injective hulls, Pacific J. Math. 25 (1968), 331-340.

24. I. R. Reiner, Maximal orders, Academic Press, London and New York, 1975.

25. O. F. G. Schilling, Noncommutative valuations, Bull. Amer. Math. Soc. 51 (1945), 297-304.

26. __, The theory of valuations, Math. Surveys, no. 4, Amer. Math. Soc., Providence, R.I., 1950.

27. M. Schröder, Angeordnete Schiefkörper mit natürlicher Bewertung vom Rang 1, Dissertation, Münster, 1985.

28. A. R. Wadsworth, Extending valuations to finite dimensional division algebras, Proc. Amer. Math. Soc. 98 (1986), 20-22.

Department of Mathematics, University of Alberta, Edmonton, Alberta, Canada

Institut für Algebra und Zahlentheorie, Technische Universität, D-3300 BraunSCHWEIG, WEST GERMANY 